\title{
TERMOS DE USO E POLÍTICAS DE PRIVACIDADE DAS REDES SOCIAIS ON-LINE
}

\section{TERMS OF USE AND PRIVACY POLICIES FOR ON-LINE SOCIAL NETWORKS}

\author{
Daniela Assis Alves Ferreiraa \\ Marta Macedo Kerr Pinheirob \\ Rodrigo Moreno Marques ${ }^{c}$
}

\begin{abstract}
RESUMO
Objetivo: Inserido no debate contemporâneo sobre privacidade de dados pessoais e políticas de informação, o presente artigo objetiva discutir como as publicações científicas brasileiras têm abordado os termos de uso e as políticas de privacidade das redes sociais on-line. Adicionalmente, objetiva identificar quais aspectos têm sido abordados na literatura acadêmica, em relação a esses termos de uso e respectivas políticas de privacidade. Metodologia: Foi realizado um estudo exploratório, analíticodescritivo, com revisão sistemática da literatura de artigos publicados em periódicos brasileiros e anais de congressos disponíveis no Portal Capes. Foram selecionados artigos que adotam os termos de uso e políticas de privacidade das redes sociais online como objeto de estudo. Resultados: A partir desse corpus, constatou-se que o tema privacidade de dados pessoais em redes sociais on-line tem sido abordado sob diversos aspectos, e principalmente em relação ao tratamento do legado digital. Conclusões: Esse tema tem alcançado crescente destaque e tem se mostrado recorrente, relevante e atual, provavelmente devido ao grande volume de dados capturados e armazenados pelos provedores de aplicações.
\end{abstract}

Descritores: Privacidade. Dados pessoais. Termos de Uso. Políticas de Privacidade. Redes Sociais on-line.

a Doutoranda do Programa de Pós-Graduação em Sistemas de Informação e Gestão de Conhecimento da Universidade Fundação Mineira de Educação e Cultura (FUMEC). E-mail: daniassis@gmail.com

b Doutora em Ciência da Informação pelo Instituto Brasileiro de Informação em Ciência e Tecnologia (IBICT) Universidade Federal do Rio de Janeiro (UFRJ). Docente da Universidade Fundação Mineira de Educação e Cultura (FUMEC) e docente colaboradora do Programa de Pós-graduação em Gestão e Organização do Conhecimento da Universidade Federal de Minas Gerais (UFMG). E-mail: martakerr@gmail.com

c Doutor em Ciência da Informação pela Escola de Ciência da Informação (ECI) da Universidade Federal de Minas Gerais (UFMG). Docente do Programa de Pós-Graduação em Ciência da Informação na Escola de Ciência da Informação (ECI) da Universidade Federal de Minas Gerais (UFMG). E-mail: rodrigomorenomarques@yahoo.com.br 


\section{INTRODUÇÃO}

O avanço das Tecnologias da Informação e Comunicação trouxe novas possibilidades de interação, novas tecnologias de conexão e novas formas de relacionamento entre as pessoas. A sociedade civil tem se mostrado preocupada com a proteção dos dados pessoais e com os riscos do uso de informações particulares por empresas e governos, tanto para comercialização de dados sobre hábitos de consumo quanto para a prática de vigilância. Assim, questões sobre a privacidade de dados surgem como um novo desafio diante dos avanços tecnológicos, sendo que esse é um tema candente nas discussões atuais e tem sido abordado sob diversos aspectos, sejam eles jurídicos, éticos, sociais, tecnológicos e das áreas da saúde, segurança, gestão, artes e humanidades.

A importância em relação ao tratamento de dados pessoais tem aumentado à medida que seu valor financeiro tem sido reconhecido por empresas que os coletam, armazenam e o utilizam como ativos empresariais. Ao analisar a necessidade de consentimento dos usuários para que empresas manipulem dados pessoais, Luger, Moran e Rodden (2013, p. 2695, tradução nossa) alegam que a "habilidade para gerenciar essa informação é o meio primordial por meio do qual [usuários] protegem sua privacidade e sua identidade"'. No entanto, completam os autores, os documentos que estabelecem os termos de consentimento não recebem o destaque que deveriam, padecendo de problemas de concepção e acessibilidade.

Dessa forma, a questão principal [QP] que esse artigo visa investigar, por meio de uma revisão sistemática de literatura (RSL), é: Como as publicações científicas têm abordado os termos de uso e os termos de serviço das redes sociais on-line? Da questão principal [QP], duas outras questões específicas $[Q E]$ foram derivadas:

[QE1]: Que estudos abordam a privacidade de dados pessoais em relação aos termos de uso e os termos de serviço das redes sociais on-line?

\footnotetext{
${ }^{1}$ No original: "ability to manage that information at the point of consent is the primary means by which we protect both our privacy and our identity".
} 
[QE2]: Quais aspectos têm sido abordados sobre termos de uso e os termos de serviço nas publicações sobre as redes sociais on-line?

A próxima seção discute os conceitos de termos de uso e políticas de privacidade das redes sociais on-line. A seguir, é apresentada a metodologia adotada para seleção das publicações analisadas na revisão sistemática de literatura. Na sequência, são destacados os estudos relacionados à temática onde se hibridam os temas: termos de uso, termos de privacidade e redes sociais. Por fim, é apresentada a análise dos dados coletados e as conclusões do trabalho.

\section{TERMOS DE USO E POLÍTICAS DE PRIVACIDADE DAS REDES SOCIAIS ON-LINE}

As redes sociais são formadas por pessoas que se reúnem por um interesse comum e que proporcionam a interação social, originária da necessidade humana de se relacionar com pares, fenômeno sempre existente na socialização entre humanos. A novidade fica por conta da tecnologia da Internet que disponibilizou recursos em plataformas diversas nas interfaces de funcionamento das redes (BARBOSA, 2014).

Nesse sentido, Meira, Soares e Pires (2012) afirmam que "a maioria dos sites de relacionamento é baseada nos perfis dos usuários, onde podem ser compartilhados os gostos e preferências de cada um. Em alguns sites, o usuário ainda tem a possibilidade de controlar a privacidade, protegendo informações do perfil". De acordo com o Instituto Nacional de Tecnologias da Comunicação (INTECO) e a Agência Espanhola de Proteção de Dados (AEPD),

As redes sociais on-line são serviços prestados por meio da Internet que permitem aos usuários gerar um perfil público, no qual captura dados pessoais e informações de si mesmos, tendo ferramentas que permitam interagir com o resto dos usuários relacionados ou não ao perfil publicado² (INTECO; AEPD, 2008, p. 7, tradução nossa).

\footnotetext{
2 No original "Las redes sociales online son servicios prestados a través de Internet que permiten a los usuarios generar un perfil público, en el que plasmar datos personales e información de uno mismo, disponiendo de herramientas que permiten interactuar con el resto de usuarios afines o no al perfil publicado".
} 
As redes sociais on-line permitiram não só uma maior socialização entre as pessoas, como também a exposição da vida privada de seus membros, além de problemas em relação à proteção da privacidade digital nessas redes. Essas redes sociais on-line adotam termos de uso que estabelecem políticas de privacidade e configurações de privacidade para seus usuários. Assim, para terem acesso e utilizarem o site e/ou aplicativo on-line é preciso que o usuário forneça seu consentimento em relação ao documento apresentado como termos de uso e políticas de privacidade.

Os termos de uso são documentos jurídicos que estabelecem regras de atuação de um site e/ou aplicativo on-line e descrevem a forma como será a interação do usuário com o serviço ofertado. Também estabelecem os direitos e deveres do usuário, assim como dos prestadores de serviço, sendo uma medida protetiva para os usuários. No entanto, a grande utilização de redes sociais on-line e sites de compartilhamento de arquivos virtuais fez com que 0 direito à privacidade de dados pessoais estivesse em risco, uma vez que a maioria dos usuários não lê esses termos e não tem ciência sobre o quanto estão expondo seus dados pessoais (BARBOSA, 2014).

Muitas vezes as políticas de privacidade são o único meio de informação para que os usuários tomem decisões. No entanto, conforme alertam Jensen e Potts (2004), há problemas com as políticas de privacidade quanto à falta de padronização, a linguagem utilizada e a incompatibilidade entre o que é publicado e o que os usuários querem saber.

Luger, Moran e Rodden (2013) também afirmam que esses termos costumam ser complexos e incompreensíveis, sendo fundamental a inclusão, acessibilidade e a clareza dos termos e condições dos principais serviços digitais.

Portanto, o ato de marcar a caixa para indicar que um usuário aceita os termos e condições de serviço deve ser baseado em uma compreensão clara do que exatamente está concordando. Sem esse entendimento, não se pode dizer verdadeiramente que o usuário está informado (LUGER; MORAN; RODDEN, 2013, p. 2688, tradução nossa). ${ }^{3}$

\footnotetext{
${ }^{3}$ No original: "Therefore, the act of ticking the box to indicate that a user accepts the terms and conditions of service must be based upon a clear comprehension of what precisely it is they are agreeing to. Without this understanding, it cannot truly be said that the user is informed".
} 
Lima (2014) compara os termos de uso a contratos de adesão eletrônicos, e alerta que eles são muito longos, repetitivos e de difícil entendimento, fazendo com que não sejam lidos na íntegra pelos usuários. Assim, ao concordarem com esses termos, os usuários se submetem integralmente às suas cláusulas, que foram elaboradas unilateralmente pelos fornecedores, reiterando a chamada "ditadura dos contratos de adesão eletrônicos". Baggio (2012) corrobora esse pensamento ao afirmar que, no momento que o usuário aceita os termos de uso e as políticas de privacidade, ele não tem conhecimento suficiente do que está contratando, e simplesmente concorda com o contrato ofertado sem se aprofundar nas informações apresentadas. "São de adesão todas as relações contratuais, nas quais a uma das partes apenas resta concordar com o conteúdo do contrato, não lhe sendo possível discuti-lo" (BAGGIO, 2012, p. 58).

Para Souza e Maciel (2015), no caso de usuários de aplicativos móveis, os usuários desconhecem não só a que riscos estão expostos quanto à captura de seus dados, como em relação aos termos de uso e a política de privacidade, que são longos para serem lidos em smartphones, de difícil compreensão e não são flexíveis para se adequarem às preferências dos usuários. Ademais, ainda que o usuário tenha tempo disponível para tentar interpretar os longos e complexos termos de uso e tenha capacidade de compreendê-los, nota-se que não resta outra opção a ele senão aceitar as condições ali impostas, caso decida usar o serviço ofertado. Portanto, o usuário se vê obrigado a aceitar na íntegra os termos de uso e permitir o acesso aos seus dados para poder utilizar os recursos de tais aplicativos.

Para maiores esclarecimentos sobre o tema, a seguir será apresentada a metodologia utilizada para a realização dessa pesquisa.

\section{METODOLOGIA}

Este artigo realizou uma revisão sistemática da literatura (RSL) sobre os termos de uso e os termos de serviço das redes sociais on-line, visando verificar como as publicações científicas abordam essa temática. A RSL foi feita por meio do levantamento de publicações no Portal Capes, seguida por 
uma análise qualitativa dos artigos selecionados. Segundo Kitchenham (2004, p. 1, tradução nossa), "uma revisão sistemática da literatura é um meio de identificar, avaliar e interpretar todas as pesquisas disponíveis relevantes para uma determinada questão de pesquisa, ou área de tema, ou fenômeno de interesse"4. A RSL é um método confiável, que sintetiza o que já foi pesquisado sobre o tema e que busca identificar lacunas existentes.

Assim, em um primeiro momento foi feito o acesso ao Portal de Periódicos da Capes, uma biblioteca virtual que permite acesso a mais de 45 mil publicações periódicas e 130 bases referenciais, nacionais e internacionais. Para alcançar um número maior de publicações, foi feito acesso remoto ao conteúdo assinado do Portal de Periódicos da Capes via Comunidade Acadêmica Federada (CAFe). ${ }^{5} \mathrm{~A}$ busca no Portal Capes para a realização da revisão sistemática foi efetuada no período de 26 de novembro a 8 de dezembro de 2019. Para a execução do estudo, primeiramente foram definidas as palavras-chave da pesquisa a partir dos possíveis termos que poderiam estar relacionados ao tema principal, sendo: "Termos de serviço", "Termos de uso", e "redes sociais". Optou-se por selecionar Atas de Congressos e Artigos de periódicos científicos para serem analisados.

A primeira busca utilizou como string de busca as expressões exatas em qualquer campo e em qualquer data de publicação: "termos de serviço" OU "termos de uso". A busca retornou 67 resultados e, após selecionar o campo "Expandir meus resultados", ampliou para 100 resultados. Foram selecionadas as opções "Atas de Congressos", com nove resultados, e "Artigos", com 68 resultados, totalizando 77 publicações a serem analisadas.

Após a leitura de todo conteúdo para verificar se as publicações atendiam às questões de pesquisa da revisão sistemática da literatura, foram selecionadas apenas as publicações que abordavam especificamente os

\footnotetext{
4 No original: "A systematic literature review is a means of identifying, evaluating and interpreting all available research relevant to a particular research question, or topic area, or phenomenon of interest".

${ }^{5}$ A Comunidade Acadêmica Federada (CAFe) é uma rede formada por instituições de ensino e pesquisa no Brasil e gerida pela Rede Nacional de Ensino e Pesquisa (RNP). O acesso remoto permite visualizar o conteúdo assinado do Portal de Periódicos disponível para cada instituição. No caso, o acesso foi feito por meio da rede da Universidade Federal de Minas Gerais.
} 
termos de uso e políticas de privacidade das redes sociais on-line. Assim, as publicações associadas a outros escopos que não os termos de uso e políticas de privacidade foram excluídas.

Excluídos os trabalhos que não atendiam aos objetivos da pesquisa, restaram nove artigos, sendo seis artigos referentes às Atas de Congressos e três artigos de periódicos científicos. Estes foram lidos na íntegra e seus conteúdos apresentados de forma compilada no próximo tópico.

A segunda busca utilizou como string as expressões exatas em qualquer campo e em qualquer data de publicação: "termos de serviço" E "redes sociais". A busca retornou um resultado e, após selecionar o campo "Expandir meus resultados", ampliou para quatro resultados. A opção "Artigos" apresentou três resultados, que foram analisados. Um dos três artigos foi descartado por ter aparecido no resultado da busca anterior e já ter sido identificado que as expressões de busca estavam presentes na publicação, porém associadas a outro escopo que não o estudado. Após a leitura dos outros dois artigos, eles foram classificados de acordo com os critérios de inclusão estabelecidos.

A terceira e última busca utilizou como string de busca as expressões exatas em qualquer campo e em qualquer data de publicação: "termos de uso" E "redes sociais". A busca retornou 15 resultados e, após selecionar o campo "Expandir meus resultados", ampliou para 30 resultados. A opção "Artigos" apresentou 21 resultados, e "Atas de Congressos", seis resultados, totalizando 27 publicações a serem analisadas. No entanto, 23 resultados da busca retornaram artigos idênticos aos já analisados na primeira e na segunda busca. Somente quatro artigos apareceram pela primeira vez nos resultados das buscas. Esses foram analisados e as expressões de busca estavam presentes nas publicações, porém, associadas a outro escopo que não o estudado. Assim, a terceira busca não apresentou nenhum artigo novo.

\section{RESULTADOS DA RSL}

Após a leitura completa dos artigos selecionados, conforme os critérios explicados anteriormente, as questões específicas de pesquisa puderam ser 
respondidas, conforme passamos a apresentar.

\section{1 [QE1]: Que Estudos Abordam a Privacidade de Dados Pessoais em Relação aos Termos de Uso e os Termos de Serviço das Redes Sociais ON-LINE?}

Viana et al. (2017) pesquisaram como algumas redes sociais tratam a morte do usuário por meio da análise dos regulamentos dessas redes sociais no Brasil. Os autores fizeram uma análise documental dos termos de uso e a política de privacidade das redes sociais escolhidas, a saber: Facebook, Pinterest, Instagram, Foursquare/Swarm, Linkedin, Whatsapp e YouTube. Os autores chamaram de "bens digitais" os dados e informações dos usuários que são registrados nas redes sociais on-line, considerando-os passíveis de serem alcançados pelos instrumentos jurídicos que regem o direito de herança. Eles verificaram, nas referidas redes sociais, a possibilidade de excluir a conta, tratar os bens digitais, cadastrar um herdeiro e o funcionamento da conta após o falecimento do proprietário. Os autores concluíram que algumas redes sociais tratam de maneira diferenciada os bens digitais dos usuários em suas políticas de privacidade, e apenas YouTube e Facebook repassavam essas informações a terceiros. Adicionalmente, argumentam que algumas estratégias do Facebook ditam muitos comportamentos adotados por redes sociais menores. Por fim, afirmam que todas as redes sociais analisadas têm diretrizes voltadas para a privacidade dos usuários e permitem que os dados sejam excluídos em caso de notificação da morte do usuário, com exceção do WhatsApp que não armazena mensagens do usuário. Apesar de o WhatsApp não armazenar mensagens, essa aplicação armazena alguns dados do usuário: modelo do smartphone usado, incluindo as versões do aplicativo e do sistema operacional; mensagem de status e foto de perfil; todos os números dos seus contatos (sem nomes); todos os grupos que o usuário participa; dados da criação d conta; definições de privacidade; recibo de leitura (tique duplo azul) e "última vez online"; e contatos bloqueados (GHEDIN, 2018).

Yamauchi, Maciel e Pereira (2018) identificaram como os usuários utilizam sistemas de pré-gerenciamento do legado digital. Após a realização de uma revisão da literatura, foi feita uma análise qualitativa e quantitativa de um 
questionário com 23 questões respondido por 180 pessoas. Na primeira parte, o questionário identificou informações pessoais dos respondentes e se sabiam o conceito de legado digital. Em caso afirmativo, o participante segue para a segunda parte, mais específica sobre sistemas de pré-gerenciamento do legado digital. Ou autores perceberam que muitos participantes não tinham conhecimento sobre legado digital, mesmo utilizando sistemas que permitam a transferência de dados após o falecimento do usuário, assim como os mesmos não pensam em configurar o destino dos seus dados. Quanto ao entendimento sobre os termos de uso e políticas de privacidade dos sistemas, apenas $4 \%$ dos participantes afirmaram que leem as cláusulas dos aplicativos, enquanto $44 \%$ afirmaram que leem às vezes e $52 \%$ não leem. Os autores concluíram então que os dados digitais têm não só crescido em volume, como também em valor ao se tornar um legado. Assim, é necessária uma maior distinção entre os tipos de sistemas de pré-gerenciamento do legado digital.

Campos et al. (2017) investigaram os memoriais digitais do Facebook em busca de soluções de especialistas e participantes de grupos focais, com base nos questionamentos sobre os elementos da web social. Quando o algoritmo do Facebook identifica que o usuário faleceu, o seu perfil se transforma em um memorial digital, a menos que seja solicitado 0 cancelamento da conta por familiares ou pelo contato herdeiro indicado pelo proprietário do perfil em suas preferências da conta do aplicativo. De acordo com a visão de especialistas, os termos de uso deveriam explicitar melhor para os usuários como eles podem escolher a destinação e/ou como se dará o gerenciamento do seu perfil após a sua morte. Os autores concluíram que muitas são as soluções para os memoriais digitais do Facebook em relação aos elementos da web social dadas por especialistas e participantes de grupos focais. Portanto, "a modelagem dos aspectos sócio técnicos em memoriais digitais é um desafio, permeado pelas questões legais, culturais, comunicacionais, de facilidade de uso e pelos valores humanos imbricados nos sistemas" (CAMPOS et al., 2017).

Leitão et al. (2017) apresentaram a visão de oito participantes da comunidade brasileira de Interação Humano-Computador (IHC) sobre o que é 
preciso para avançar no escopo do Desafio 4 - Valores Humanos, que engloba questões relacionadas à privacidade, legado digital pós-morte, ética e designt. Ainda são poucos os trabalhos que tratam sobre o tema valores humanos entre os trabalhos publicados na principal conferência de IHC do Brasil. Implementar ações estratégicas para fomentar a interdisciplinaridade é um desafio, uma vez que a maior parte dessa comunidade é formada por pesquisadores da ciência da computação, o que mostra a necessidade de interagir com profissionais das diferentes áreas das ciências humanas e sociais. É preciso articular uma discussão mais global em torno do Desafio 4 - Valores Humanos, sendo a interdisciplinaridade o caminho a ser seguido. Os estudos indicaram a necessidade de criação "de uma agenda de pesquisa que enfatize a ética e a interdisciplinaridade na abordagem do Desafio 4: Valores Humanos, e que se dedique a buscar respostas para as questões apresentadas nesta seção" (LEITÃO et al., 2017).

Parchen e Freitas (2016) realizaram uma pesquisa explicativa, que também foi exploratória e descritiva, que teve como objetivo discutir questões ligadas ao espólio de dados e informações digitais disponíveis em tecnologias atuais, tais como as redes sociais e a computação em nuvem. Também discutiu questões legais sobre o patrimônio digital, quando do falecimento do titular, a ser transferido aos herdeiros ou se passa a ser do provedor de produtos e serviços, suscitando essa complexa questão e necessária discussão na atualidade diante do grande volume de dados e informações que circulam na Internet. Os autores discorrem sobre o alto valor econômico que dados e informações possuem no mercado, assim como a expropriação dos direitos dos usuários de produtos e serviços ofertados na Internet.

Os termos de uso dos serviços on-line mantêm a propriedade intelectual dos dados dos usuários, mas transferem os direitos de administração sobre o conteúdo produzido para os fornecedores de aplicações. Isso justifica o fato das empresas que oferecem serviços on-line de "forma gratuita", tais como

\footnotetext{
${ }^{6}$ A comunidade brasileira de Interação Humano-Computador (IHC) estabeleceu cinco Grandes Desafios de Pesquisa em IHC no Brasil (GranDIHC-BR) para o período de 2012 a 2022, a saber: 1. Futuro, Cidades Inteligentes e Sustentabilidade; 2. Acessibilidade e Inclusão Digital; 3. Ubiquidade, Múltiplos Dispositivos e Tangibilidade; 4. Valores Humanos; 5. Formação em IHC e Mercado (BARANAUSKAS; SOUZA; PEREIRA, 2014).
} 
Google e Facebook, terem se tornado tão valiosas, uma vez que repassam os dados de seus usuários para anunciantes e/ou desenvolvedores de novas aplicações. Além disso, esses serviços não se responsabilizam por perdas ou danos nos dados dos seus usuários, assim como deixam claro que irão capturar informações associadas ao usuário, mesmo após a exclusão de sua conta, se assim julgarem necessário, além da venda de informações para otimizarem os anúncios exibidos. Ou seja, os provedores de serviços on-line deixam claro que os conteúdos postados e/ou transmitidos em suas plataformas não serão utilizados, mas sim o seu comportamento será analisado, gerando informações sobre as quais o usuário não tem poder de administrar. Assim os autores concluem que, diante do valor da informação, é necessário regulamentar a propriedade sobre esses dados presentes nos serviços on-line, podendo gerar a arrecadação de impostos ao serem transmitidos aos herdeiros quando do falecimento do titular.

Yamauchi, Souza e Silva Junior (2016) discutiram sobre os termos de uso e políticas de privacidade de aplicativos móveis. Em um primeiro momento, foi realizada uma revisão sistemática para busca e seleção de estudos sobre a privacidade dos usuários em projetos de sistemas ubíquos. Assim, os autores apresentaram dez diretrizes para auxiliar os desenvolvedores desses sistemas em relação ao estabelecimento da confiança e da privacidade, a saber:

1. Usar linguagem clara;

2. Permitir checagem e liberdade de configuração;

3. Possuir clara finalidade de uso de dados;

4. Realizar coleta mínima de dados;

5. Permitir controle e transparência de dados;

6. Utilizar mecanismos de segurança;

7. Obedecer a legislação vigente;

8. Flexibilizar os termos de uso e políticas de privacidade;

9. Notificar o usuário quando houver alteração em algum termo; 10. Disponibilizar os termos no idioma do aplicativo (YAMAUCHI; SOUZA; SILVA JUNIOR, 2016, p. 2).

Em relação ao foco específico do trabalho apresentado, sobre a questão da privacidade dos termos de uso e políticas de privacidade, os autores deram destaque aos itens de sete a dez. Quanto a obedecer à legislação vigente, recomenda-se que o aplicativo atenda às legislações vigentes no país em que está sendo utilizado, pois isso geraria mais confiança e segurança aos 
usuários. A flexibilização dos termos de uso e políticas de privacidade deveria permitir aos usuários a escolha de quais recursos do smartphone seriam utilizados pelo aplicativo, uma vez que o acesso a essas ferramentas não fossem imprescindíveis para o funcionamento do aplicativo. O usuário deveria ser sempre notificado toda vez que ocorresse alguma alteração e/ou atualização no termo de uso e/ou na política de privacidade do aplicativo, destacando a mudança de uma forma comparativa do texto anterior diante ao atual. Por fim, esses termos de uso e políticas de privacidade deveriam ser apresentados sempre no idioma do país em que o aplicativo está sendo utilizado para serem mais bem compreendidos pelos usuários. Assim, os autores utilizaram as quatro últimas diretrizes para analisar o aplicativo Waze como exemplo para levantamento de problemas e indicações de possíveis soluções.

Esse aplicativo apresenta as interfaces em português, mas os termos de uso e a política de privacidade estão em inglês e são muito extensos, o que dificulta a compreensão dos usuários. O aplicativo também oferece as opções de utilização com e sem login, o que dá uma falsa sensação de controle de privacidade dos dados do usuário ao optar pela utilização sem login. No entanto, não oferece a flexibilidade de aceitar parcialmente o Contrato de Licença de Usuário Final, que contempla o termo de uso e a política de privacidade. Ou o usuário aceita o contrato ou não consegue acessar 0 aplicativo. O Waze não obedece à legislação brasileira vigente e segue as leis do Estado de Israel, o que dificulta o questionamento judicial em relação aos termos de uso e fere o artigo 8 do Marco Civil da Internet, que determina a nulidade de contratos de adesão que não ofereçam o foro brasileiro como opção (BRASIL, 2014). Além disso, o aplicativo não informa por quanto tempo irá reter os dados dos usuários, em caso de exclusão da conta, mas informa que os dados poderão ser compartilhados com outras empresas. Os autores acreditam que, a partir da adoção de práticas de respeito a privacidade, os usuários de aplicativos móveis irão se preocupar mais com o direito sobre seus dados pessoais.

Silva et al. (2018) analisaram como a privacidade tem sido tratada nas 
pesquisas de Interação Humano-Computador brasileiras, para observar como o quarto desafio, sobre privacidade e a visibilidade da informação do usuário, publicado no relatório Brasil Great Challenges of Research in HCl (I GranDIHC$\mathrm{BR}$ ) tem colaborado com o desenvolvimento dessa temática nas dez edições do Simpósio Brasileiro de Fatores Humanos em Sistemas Computacionais (IHC Brasil). Foram mapeados 314 artigos sobre privacidade publicados nas últimas dez edições do IHC Brasil, nas cinco edições anteriores e nas cinco edições posteriores ao I GranDIHC-BR, valendo-se de uma revisão sistemática da literatura com abordagem comparativa. Foram identificados poucos artigos relacionados à privacidade e visibilidade de informações sobre os usuários. Em relação aos termos de uso e políticas de privacidade, os autores encontraram somente o artigo já citado de Yamauchi, Souza e Silva Junior (2016). Esses achados demonstram que ainda há muitos desafios sobre o assunto a serem investigados pela comunidade da IHC.

Almeida e Almeida (2016) fizeram uma análise dos termos de uso e as políticas de privacidade de alguns provedores de aplicação de Internet sob a luz do princípio da finalidade e a cooperação destes provedores com as agências de inteligência. Para tal, foi feita uma pesquisa bibliográfica e documental, seguida de uma análise qualitativa dos termos de uso do Pokémon Go, Google e Facebook. Os autores estudaram esses provedores de aplicação de internet frente ao Código de Defesa do Consumidor, o Código Civil e o Marco Civil da Internet, e identificaram que seus termos de uso e políticas de privacidade regulam o tratamento dos dados pessoais, devendo atender ao princípio da finalidade. A pesquisa mostrou que, mediante requisição judicial, existe a obrigatoriedade de os provedores de aplicações de internet sediados nos EUA cooperarem com agências de inteligência, mesmo que nos termos de uso e políticas de privacidade isso não seja explicitado de maneira clara. Apesar de os termos de uso e políticas de privacidade dos provedores de aplicações de internet estudados não deixarem claro como e em que circunstâncias os dados pessoais podem ser compartilhados com a agência de inteligência, isso pode ocorrer caso haja possibilidade de violação da segurança de outros usuários, dos próprios provedores ou da sociedade em 
geral. Esses provedores de aplicações de internet são obrigados a cooperarem com agências de inteligência dos EUA, uma vez que lá estão situados. Mas no Brasil, essas empresas devem seguir o Marco Civil da Internet e os dados dos usuários só são compartilhados mediante determinação judicial e/ou para as finalidades definidas nos seus termos de uso e políticas de privacidade. Eles precisam atender a finalidade da coleta e compartilhamento dos dados pessoais definidas nos termos de uso e políticas de finalidade, principalmente se forem cooperar com as agências de inteligência - isso precisa estar claro e explícito para os usuários.

Quadros e Marcon (2014) discutem os conceitos de público e privado nas redes sociais por meio da análise dos termos de uso e da política de privacidade do Facebook, no ano de 2014. De acordo com as autoras,

[...] para que o usuário faça sua adesão e torne-se integrante de alguma rede social é preciso fazer um cadastro com seus dados pessoais, além de autorizar os Termos de Uso e as Políticas de Privacidade desse ambiente, disponíveis em links que encaminham, na maioria das vezes, para uma nova página com textos muito extensos e minuciosos, onde constam todos os deveres e as obrigações de conduta dentro daquele ambiente (QUADROS; MARCON, 2014, p. 69).

Ao aceitar os termos de uso de uma rede social, o usuário fica vulnerável às preferências em relação à sua privacidade, pelo fato de muitos não lerem o seu real conteúdo e por optarem pela exposição de dados pessoais, fotos, opiniões e detalhes da sua vida privada. As autoras apresentam o paradoxo existente entre o público, o privado e a privacidade sob o ponto de vista da teoria da Modernidade Líquida de Zygmunt Bauman (2001; 2010; 2011) e afirmam que, quase sempre, esses termos não são lidos pelos usuários, permitindo o acesso aos seus dados pessoais e colocando em risco a sua privacidade. Por fim, concluem que nas redes sociais ocorre uma ressignificação dos conceitos de público e privado devido à necessidade de exposição e conexão dos usuários. Essas redes sociais possibilitam uma maior conexão e interação entre as pessoas, suas vidas e culturas, e é isso que sustenta as relações humanas na Internet. As autoras não acreditam que os usuários deixarão de utilizar as redes sociais ao conhecerem suas políticas e termos de uso, mas alertam para necessidade de maior atenção quanto à 
segurança e a preservação da intimidade em relação ao conteúdo publicado.

Pisa (2014) analisou, por meio da análise do discurso, as políticas de privacidade e de termos de serviço do buscador Google e sob a perspectiva de poder abordada por Michel Foucault (1975; 1988; 1999; 2006; 2007). A autora discutiu noções de poder jurídico, estratégico e disciplinar, assim como questões sobre controle, vigilância e sensação de liberdade de ação e de fala na Internet. Demonstra que os termos de serviço do Google exercem seu poder autoritário ao impor aos usuários barreiras de acesso aos seus próprios dados quando precisan recuperá-los, assim como a concessão ao Google do direito de acesso e uso, mesmo que restrito, ao seu material publicado, mesmo quando o usuário deixa de utilizar o serviço. "Assim, mesmo os termos de serviços e políticas de privacidade estarem a disposição de qualquer usuário, é por meio da disciplinarização e pelo poder estratégico que certos discursos passam a ser tomados como verdadeiros" (PISA, 2014, p. 263).

Ao utilizar os serviços da empresa Google como seu motor de busca e outras aplicações on-line, os usuários têm uma sensação de liberdade de ação e de fala na Internet, mas não percebem o controle, a vigilância e até mesmo a apropriação privada de seus dados e informações. Esse sentimento de ausência de poder que o Google exerce sobre os usuários faz com que esses últimos utilizem com confiança os serviços da empresa, que utiliza de um discurso democrático para conseguir registrar os dados de seus usuários e alimentar seu enorme banco de dados. O Google exerce um poder jurídico ao utilizar os dados dos usuários e thes dificultar acesso a esses dados armazenados. O poder disciplinar é percebido quando a empresa transforma esses dados registrados em informação e, com isso, geram conhecimento que é utilizado como forma de controle dos assuntos que devem ser veiculados e tomados como verdadeiros. Por fim, a autora conclui que o Google cria uma sensação de ausência de poder sobre o que os usuários fazem no site e nos outros sites de sua propriedade, sendo isso a sua forma de exercer um controle não democrático e gerar um discurso de poder, tendências, novos produtos e serviços em relação às informações que armazena. Sendo assim, não há liberdade de discussão, uma vez que determinados temas são manipulados 
para controlar os discursos e manipular a opinião das massas, que acreditam exercer uma liberdade de pensamento.

Também por meio de uma pesquisa explicativa, exploratória e descritiva, Freitas (2017) discutiu o tratamento de dados pessoais sob a ótica da legislação brasileira, assim como apresenta a caracterização de perfil (profiling), que é a aplicação de métodos e técnicas computacionais em dados pessoais, com o objetivo de generalizar uma população e criar uma representatividade estatística. Dentre as legislações apresentadas, a autora destaca o Anteprojeto de Lei de Proteção de Dados Pessoais (Projeto de Lei №. 5.276/2016), que aborda o tratamento de dados pessoais e a necessidade do consentimento expresso por parte do titular como uma das formas de controle do uso indiscriminado de dados pessoais, por exemplo, pelas redes sociais. Assim, a autora afirma que "é evidente a intenção do provedor do serviço ou aplicativo em capturar para si um volume de dados pessoais ou não, sendo que todos os textos, seja da política de privacidade ou dos termos de serviços, consideram que o usuário lê e compreende a complexidade dos mesmos" (FREITAS, 2017, p. 26). A autora ainda alerta que, além de não possuir conhecimento técnico para entender o conteúdo, os usuários raramente leem os extensos termos e políticas das empresas provedoras de serviços e aplicativos digitais. É necessário divulgar os riscos existentes ao aceitar as condições oferecidas por essas empresas em relação ao tratamento de seus dados pessoais, assim como a necessidade de discutir os aspectos técnicos e jurídicos em relação ao tratamento desses dados. Uma vez que clicaram em aceitar, tendo lido ou não os termos de uso e políticas de privacidade, os usuários autorizaram essas empresas a compartilharem seus dados. Esse tratamento de dados pessoais pode criar discriminação sob a ótica da legislação brasileira, mas nem sempre isso pode ser percebido pelos usuários.

\section{2 [QE2]: QuaIS AsPectos TÊm Sido Abordados Sobre os TeRmos de USO E os Termos de Serviço nas Publicações Sobre as Redes Sociais ON-Line?}

Para facilitar a visualização das respostas encontradas para a [QE2], o Quadro 1 apresenta os autores agrupados conforme os temas pesquisados e 
as principais conclusões dos artigos recuperados por meio da RSL.

\section{Quadro 1 - Síntese dos artigos analisados}

\begin{tabular}{|c|c|c|}
\hline Autores & Tema de pesquisa & $\begin{array}{l}\text { Principais conclusões } \\
\end{array}$ \\
\hline $\begin{array}{l}\text { Viana, Maciel, } \\
\text { Arruda e Souza } \\
\text { (2017) } \\
\text { Yamauchi, Maciel } \\
\text { e Pereira (2018) } \\
\text { Campos, Justi, } \\
\text { Maciel e Pereira } \\
\text { (2017) } \\
\text { Leitão, Maciel, } \\
\text { Piccolo, Salgado, } \\
\text { Souza, Prates, } \\
\text { Pereira, Pereira } \\
\text { (2017) } \\
\text { Parchen e Freitas } \\
\text { (2016) }\end{array}$ & $\begin{array}{l}\text { Tratamento do } \\
\text { legado digital }\end{array}$ & $\begin{array}{l}\text { - Diante do aumento de dados dos usuários, o } \\
\text { legado digital possui grande valor econômico e } \\
\text { deve ser tratado como um patrimônio rentável, } \\
\text { passível de ser transferido para herdeiros quando } \\
\text { do falecimento do proprietário e render a cobrança } \\
\text { de tributos sobre esses bens digitais. } \\
\text { - Termos de uso e políticas de privacidade de } \\
\text { aplicativos são mal redigidos e inadequados para } \\
\text { serem lidos em dispositivos móveis, pouco ou } \\
\text { nada flexíveis, e muitas vezes não estão em } \\
\text { conformidade com a legislação vigente. } \\
\text { - Nem todas as redes sociais analisadas seguem as } \\
\text { recomendações sobre tratamento do legado digital } \\
\text { e recomendam confrontar esses regulamentos } \\
\text { com a legislação brasileira sobre privacidade de } \\
\text { dados. } \\
\text { - Os termos de uso deveriam explicitar melhor para } \\
\text { os usuários como eles podem escolher a } \\
\text { destinação e/ou como se dará o gerenciamento de } \\
\text { seu perfil após a sua morte. } \\
\text { - Os termos de uso dos serviços on-line preservam } \\
\text { o direito de propriedade intelectual dos dados dos } \\
\text { usuários, mas o direito de administração desses } \\
\text { conteúdos é apropriado pelos fornecedores } \\
\text { dessas tecnologias. } \\
\text { - Questões legais, culturais, comunicacionais, de } \\
\text { facilidade de uso e dos valores humanos são } \\
\text { temas que deveriam ser abordados com uma } \\
\text { perspectiva interdisciplinar, tanto de sua definição } \\
\text { como também de sua solução, devendo estimular } \\
\text { a participação de profissionais de outras áreas, } \\
\text { principalmente pesquisadores das ciências } \\
\text { humanas e sociais. }\end{array}$ \\
\hline $\begin{array}{l}\text { Yamauchi, Souza } \\
\text { e Silva Junior } \\
\text { (2016) } \\
\text { Silva, Torres, } \\
\text { Sacramento, } \\
\text { Capra, Ferreira e } \\
\text { Garcia (2018) } \\
\text { Almeida e Almeida } \\
\text { (2016) } \\
\text { Quadros e Marcon } \\
\text { (2014) }\end{array}$ & Privacidade & $\begin{array}{l}\text { - Os desenvolvedores de sistemas devem estar } \\
\text { atentos em relação à confiança e privacidade } \\
\text { oferecida pelos aplicativos, que não devem tornar } \\
\text { o usuário inseguro, mas deve orientá-lo para um } \\
\text { uso consciente. } \\
\text { - É importante o consentimento e a compreensão } \\
\text { do usuário quanto a seus dados compartilhados. } \\
\text { - Os termos de uso e políticas de privacidade são } \\
\text { contratos de adesão que o usuário aceita para } \\
\text { utilizar os serviços dos provedores de aplicação } \\
\text { de internet, sendo assim, importante que as } \\
\text { cláusulas sejam claras sobre a coleta e o } \\
\text { tratamento de dados dos usuários. } \\
\text { - É preciso alertar os usuários de redes sociais } \\
\text { quanto à necessidade de preservar a sua } \\
\text { intimidade, mesmo sabendo que estes não vão } \\
\text { deixar de utilizar essas aplicações ao tomarem } \\
\text { conhecimento do que está definido em seus } \\
\text { termos de uso e políticas de privacidade. }\end{array}$ \\
\hline
\end{tabular}




\begin{tabular}{|c|c|c|}
\hline Pisa (2014) & $\begin{array}{l}\text { Controle e } \\
\text { vigilância }\end{array}$ & $\begin{array}{l}\text { - Os dados registrados dos usuários são } \\
\text { transformados em informação e, com isso, geram } \\
\text { conhecimento, que é utilizado como uma forma de } \\
\text { controle dos assuntos que devem ser veiculados e } \\
\text { tomados como verdadeiros. } \\
\text { - Esse novo conhecimento gerado a partir dos } \\
\text { dados coletados e compilados em informações se } \\
\text { transforma em novos produtos, novos serviços, } \\
\text { novos modos de ser e novos discursos a serem } \\
\text { ofertados, o que representa a dinâmica de poder } \\
\text { disciplinador almejada pela empresa. }\end{array}$ \\
\hline Freitas (2017) & $\begin{array}{c}\text { Tratamento de } \\
\text { dados pessoais e a } \\
\text { caracterização de } \\
\text { perfil (profiling). }\end{array}$ & $\begin{array}{l}\text { - A caracterização de perfil (profiling) pode criar } \\
\text { discriminação sob a ótica da legislação brasileira, } \\
\text { mas nem sempre isso pode ser percebido pelos } \\
\text { usuários. } \\
\text { - Os usuários raramente leem os termos de uso e } \\
\text { políticas de privacidade dos provedores de } \\
\text { serviços e aplicativos digitais, mas, ao aceitarem, } \\
\text { autorizaram essas empresas a compartilharem } \\
\text { seus dados. } \\
\text { - É necessário discutir os aspectos técnicos e } \\
\text { jurídicos em relação ao tratamento de dados } \\
\text { pessoais, assim como divulgar os riscos } \\
\text { existentes em aceitar as condições oferecidas por } \\
\text { essas empresas em relação a isso. }\end{array}$ \\
\hline
\end{tabular}

Fonte: Dados da Pesquisa (2020).

Conforme o Quadro 1, dos 11 artigos selecionados, cinco artigos pesquisaram como é feito o tratamento do legado digital em caso de morte do usuário, quatro artigos abordaram questões ligadas à privacidade, um estudou questões relacionadas ao controle e vigilância nas redes sociais on-line, e, por último, um artigo abordou o tratamento de dados pessoais e a caracterização de perfil (profiling).

Assim, foi possível perceber que o tema privacidade de dados pessoais em redes sociais on-line tem sido abordado sob aspectos diversos, mas principalmente em relação ao tratamento do legado digital. Isso se dá devido, provavelmente, ao grande volume de dados capturados e armazenados pelos provedores de aplicações, o que tem despertado muitas discussões sobre o valor econômico e o futuro do destino que será dado a esse legado digital quando seu proprietário vier a falecer.

\section{CONSIDERAÇÕES FINAIS}

Este artigo apresentou o resultado de uma Revisão Sistemática de 
Literatura (RSL), com o objetivo de discutir como as publicações científicas brasileiras têm abordado termos de uso e os termos de serviço das redes sociais on-line, bem como identificar que estudos abordam a privacidade de dados pessoais e quais aspectos têm sido abordados na literatura acadêmica em relação aos termos de uso e os termos de serviço das redes sociais on-line. Essa RSL visa atender ao objetivo geral de tese de doutorado em desenvolvimento que pretende analisar a adequação das políticas de privacidade e os termos de serviço de algumas redes sociais on-line em relação às diretrizes da Lei Geral de Proteção de Dados Pessoais (LGPD).

Foi observado que os estudos que relacionam a questão da privacidade de dados pessoais em redes sociais on-line são recentes na literatura acadêmica brasileira, principalmente ao abordar os termos de serviço ou termos de uso e as políticas de privacidade dos prestadores de serviços online. O tema privacidade de dados pessoais em redes sociais on-line tem alcançado crescente destaque, se mostrado recorrente, relevante e atual e tem sido abordado sob diversos aspectos, principalmente em relação ao tratamento do legado digital.

Várias questões sobre o tema têm sido suscitadas e debatidas diante do grande volume de dados que têm sido capturados e armazenados pelos provedores de aplicações no mundo virtual. Os termos de serviço e as políticas de privacidade dessas aplicações on-line costumam ser complexos, de difícil leitura e compreensão e, na maioria das vezes, não são lidos na íntegra pelos usuários. Muitas vezes esses termos não estão em conformidade com a legislação vigente e não são flexíveis, impondo que os usuários os aceitem integralmente para que tenham acesso aos serviços ofertados. A maior parte dos usuários não consegue compreender o quanto estão expondo sua privacidade e colocando em risco a sua segurança e a preservação de seus dados pessoais.

Um dos aspectos evidenciados pela RSL foi a destinação do conteúdo produzido pelos usuários após seu falecimento, um tema complexo e que ainda exige pesquisa e debate, uma vez que é um problema recente. Os resultados da RSL sugerem que a maior parte dos usuários das redes sociais on-line 
ainda não possui conhecimento suficiente sobre o tratamento de seu legado digital. A maioria dos usuários desconhece que esse legado pode ser transferido após o falecimento do titular da conta e que ele pode ter um valor econômico para os provedores de aplicações na Internet. Assim, os termos de uso e as políticas de privacidade dos serviços on-line deveriam explicitar mais claramente a seus usuários como fazer o gerenciamento e e/ou a destinação de seu legado digital em consonância com a legislação vigente sobre 0 tratamento de dados pessoais.

Apesar da exclusão de um número expressivo de artigos, uma vez que a seleção procurou atender às questões específicas de pesquisa, os 11 artigos que compuseram o corpus do estudo possibilitam apresentar um panorama das pesquisas sobre o tema, apresentado nesses estudos recentes. Destaca-se a necessidade de evolução de estudos que discutam um tema que tem se mostrado cada vez mais importante e recorrente no cotidiano das pessoas.

\section{REFERÊNCIAS}

ALMEIDA, Juliana Evangelista de; ALMEIDA, Daniel Evangelista Vasconcelos. Os provedores de aplicação de internet e a mitigação do princípio da finalidade em vista da cooperação com agências de inteligência. Revista de Direito, Governança e Novas Tecnologias, Curitiba, v. 2, n. 2, p. 53-74, jul./dez. 2016. Disponível em:

https://www.indexlaw.org/index.php/revistadgnt/article/view/1487. Acesso em: 30 nov. 2020.

BAGGIO, Andreza Cristina. 0 direito do consumidor brasileiro e a teoria da confiança. São Paulo: Editora Revista dos Tribunais, 2012.

BARANAUSKAS, Maria Cecilia Calani; SOUZA, Clarisse S. de; PEREIRA, Roberto. I GranDIHC-BR - Grandes Desafios de Pesquisa em Interação Humano-Computador no Brasil: Relatório Técnico. Comissão Especial de Interação Humano-Computador (CEIHC). Cuiabá: Sociedade Brasileira de Computação (SBC). 2014. Disponível em:

https://doi.org/10.13140/2.1.3651.9201. Acesso em: 30 nov. 2020.

BARBOSA, Murilo Oliveira. A importância do direito à privacidade digital, redes sociais e extensão universitária. Fragmentos de Cultura, Goiânia, v. 24, n. 8, p. 89-97, dez. 2014. Disponível em: http://dx.doi.org/10.18224/frag.v24i0.3757. Acesso em: 1 mar. 2021. 
BAUMAN, Zygmunt. Modernidade líquida. Rio de Janeiro: Jorge Zahar, 2001.

BAUMAN, Zygmunt. Capitalismo Parasitário. Rio de Janeiro, Jorge Zahar, 2010.

BAUMAN, Zygmunt. 44 cartas do mundo líquido moderno. Rio de Janeiro, Jorge Zahar, 2011.

BRASIL. Lei no 12.965, de 23 de abril de 2014. Estabelece princípios, garantias, direitos e deveres para o uso da Internet no Brasil. Brasília, DF: Presidência da República, 2014. Disponível em: http://www.planalto.gov.br/ccivil_03/_ato2011-2014/2014/lei//12965.htm. Acesso em: 28 out. 2020.

CAMPOS, Ketelem Lemos de; JUSTI, Thais; MACIEL, Cristiano; PEREIRA, Vinicius Carvalho. Digital memorials: a proposal for data management beyond life. In: BRAZILIAN SYMPOSIUM ON HUMAN FACTOR IN COMPUTING SYSTEMS, 16., 2017, Joinville. Proceedings [...]. New York: ACM, 2017. 10 p. Disponível em: https://doi.org/10.1145/3160504.3160551. Acesso em: 30 nov. 2020.

FOUCAULT, Michel. Vigiar e punir. 30. ed. Rio de Janeiro: Vozes, 1975.

FOUCAULT, Michel. História da sexualidade: a vontade de saber. Rio de Janeiro: Graal, 1988.

FOUCAULT, Michel. Microfísica do Poder. 14. ed. Rio de Janeiro: Graal, 1999.

FOUCAULT, Michel. O que é um autor. In: FOUCAULT, Michel. Estética: literatura e pintura, música e cinema. 2. ed. Rio de Janeiro: Forense Universitária, 2006.

FOUCAULT, Michel. A ordem do discurso. 15. ed. São Paulo: Edições Loyola, 2007.

FREITAS, Cinthia Obladen de Almendra. Tratamento de dados pessoais e a Legislação Brasileira frente ao Profiling e à discriminação a partir das novas tecnologias. Revista de Direito, Governança e Novas Tecnologias, Maranhão, v. 3, n. 2, p. 18-38, jul./dez. 2017. Disponível em: http://dx.doi.org/10.26668/IndexLawJournals/2526-0049/2017.v3i2.2430. Acesso em: 30 dez. 2020.

GHEDIN, Rodrigo. Quais dados o WhatsApp guarda dos usuários e como solicitar os seus. Manual do Usuário, 28 maio 2018. Disponível em: https://manualdousuario.net/whatsapp-backup-relatorio. Acesso em: 27 out. 2020. 
INSTITUTO NACIONAL DE TECNOLOGÍAS DA LA COMUNICACIÓN (INTECO); AGENCIA ESPAÑOLA DE PROTECCIÓN DE DATOS (AEPD). Estudio sobre la privacidad de los datos personales y la seguridad de la información en las redes sociales online. Madrid, feb. 2009. 159 p.

Disponível em: https://www.uv.es/limprot/boletin9/inteco.pdf. Acesso em: 1 mar. 2021.

JENSEN, Carlos; POTTS, Colin. Privacy policies as decision-making tools: an evaluation of online privacy notices. In: SIGCHI CONFERENCE ON HUMAN FACTORS IN COMPUTING SYSTEMS, 4., 2004, Vienna. Proceedings [...]. New York: ACM, apr. 2004. p. 471-478. Disponível em:

https://doi.org/10.1145/985692.985752. Acesso em: 1 mar. 2021.

KITCHENHAM, Barbara. Procedures for performing systematic reviews.

Keele: Keele University Technical Report TR/SE-0401, 2004. Disponível em: http://www.inf.ufsc.br/ aldo.vw/kitchenham.pdf. Acesso em: 1 mar. 2021.

LEITÃO, Carla; MACIEL, Cristiano; PICCOLO, Lara Schibelsky Godoy; SALGADO, Luciana; SOUZA, Patricia C. de; PRATES, Raquel; PEREIRA, Roberto; PEREIRA, Vinicius Carvalho. Human Values in $\mathrm{HCl}$ : a challenge for the GrandIHC-BR. In: BRAZILIAN SYMPOSIUM ON HUMAN FACTOR IN COMPUTING SYSTEMS, 16., 2017, Joinville. Proceedings [...]. New York: ACM, 2017. 6 p. Disponível em: https://doi.org/10.1145/3160504.3160565. Acesso em: 30 nov. 2020.

LIMA, Cíntia Rosa Pereira de. O ônus de ler o contrato no contexto da "ditadura" dos contratos de adesão eletrônicos. In: CONGRESSO NACIONAL DO CONSELHO NACIONAL DE PESQUISA E PÓS-GRADUAÇÃO EM DIREITO (CONPEDI), 23., 2014, João Pessoa. Anais [...]. Florianópolis: CONPEDI, 2014. p. 443-465. Disponível em: http://publicadireito.com.br/artigos/?cod=981322808aba8a03. Acesso em: 15 mar. 2021.

LUGER, Ewa; MORAN, Stuart; RODDEN, Tom. Consent for all: revealing the hidden complexity of terms and conditions. In: SIGCHI CONFERENCE ON HUMAN FACTORS IN COMPUTING SYSTEMS, 13., 2013, Paris.

Proceedings [...]. New York: ACM, 2013. p. 2687-2696. Disponível em: https://doi.org/10.1145/2470654.2481371. Acesso em: 1 mar. 2021.

MEIRA, Laís Moreschi de; SOARES, Matheus Fernandes de Souza; PIRES, Panmella Rodrigues. Direito à privacidade e as relações na Internet. JurisWay, 14 mar. 2012. Disponível em:

https://www.jurisway.org.br/v2/dhall.asp?id_dh=7319. Acesso em: 1 mar. 2021.

PARCHEN, Charles Emmanuel; FREITAS, Cinthia OA. Crise da Informação: a quem pertence?. Revista de Direito, Governança e Novas Tecnologias, Brasília, v. 2, n. 1, p. 22-38, jan./jun. 2016. Disponível em: 
https://www.indexlaw.org/index.php/revistadgnt/article/view/787. Acesso em: 30 dez. 2020.

PISA, Lícia Frezza. Discurso e poder em Michel Foucault: o controle do que dizemos na rede visto pela política de privacidade do Google. Domínios de Lingu@gem, Uberlândia, v. 8, n. 1, p. 250-266, jan./jun. 2014. Disponível em: https://doi.org/10.14393/DL15-v8n1a2014-14. Acesso em: 30 nov. 2020.

QUADROS, Amanda Maciel de; MARCON, Karina. Os conceitos de público e privado nas redes sociais e suas implicações pedagógicas. Revista Espaço Acadêmico, Maringá, n. 160, p. 68-77, set. 2014. Disponível em: http://periodicos.uem.br/ojs/index.php/EspacoAcademico/article/view/24485. Acesso em: 30 nov. 2020.

SILVA, Edenildo; TORRES, Bruno; SACRAMENTO, Carolina; CAPRA, Eliane Pinheiro; FERREIRA, Simone Bacellar Leal; GARCIA, Ana Cristina Bicharra. Privacy: what is the research scenario in Brazilian Symposium IHC?. In: BRAZILIAN SYMPOSIUM ON HUMAN FACTOR IN COMPUTING SYSTEMS, 17., 2018, Belém. Proceedings [...]. New York: ACM, 2018. 8 p. Disponível em: https://doi.org/10.1145/3274192.3274226. Acesso em: 30 nov. 2020.

SOUZA, Patricia C. de; MACIEL, Cristiano. Legal issues and user experience in ubiquitous systems from a privacy perspective. In: INTERNATIONAL CONFERENCE ON HUMAN ASPECTS OF INFORMATION SECURITY, PRIVACY, AND TRUST, 3., 2015, Berlim. Proceedings [...] Berlin: Springer International Publishing, 2015. p. 449-460 Disponível em: https://link.springer.com/chapter/10.1007/978-3-319-20376-8_40. Acesso em: 1 mar. 2021.

VIANA, Gabriel T.; MACIEL, Cristiano; ARRUDA, Ney A. de; SOUZA, Patricia C. de. Análise dos termos de uso e políticas de privacidade de redes sociais quanto ao tratamento da morte dos usuários. In: WORKSHOP SOBRE ASPECTOS DA INTERAÇÃO HUMANO-COMPUTADOR PARA A WEB SOCIAL (WAIHCWS), 8., 2017, Joinville. Anais [...]. Porto Alegre: Sociedade Brasileira de Computação, dez. 2017. p. 82-93. Disponível em: https://sol.sbc.org.br/index.php/waihcws/article/view/3867. Acesso em: 30 nov. 2020.

YAMAUCHI, Eduardo Akimitsu; MACIEL, Cristiano; PEREIRA, Vinícius Carvalho. An Analysis of Users' Preferences on Pre-Management of Digital Legacy. In: BRAZILIAN SYMPOSIUM ON HUMAN FACTOR IN COMPUTING SYSTEMS, 17., 2018, Belém. Proceedings [...]. New York: ACM, 2018. 5 p. Disponível em: https://doi.org/10.1145/3274192.3274237. Acesso em: 30 nov. 2020.

YAMAUCHI, Eduardo Akimitsu; SOUZA, Patricia C. de; SILVA JUNIOR, Deógenes Pereira da. Prominent issues for privacy establishment in privacy policies of mobile apps. In: BRAZILIAN SYMPOSIUM ON HUMAN FACTOR IN 


\title{
TERMS OF USE AND PRIVACY POLICIES FOR ON-LINE SOCIAL NETWORKS
}

\begin{abstract}
Objective: Inserted in the contemporary debate on personal data privacy and information policies, this article aims to discuss how Brazilian scientific publications have addressed the terms of use and privacy policies of online social networks. Additionally, it aims to identify which aspects have been addressed in the academic literature, in relation to these terms of use and respective privacy policies. Methodology: An exploratory, analytical-descriptive study was carried out, with a systematic review of the literature of articles published in Brazilian journals and conference proceedings available on the Capes Portal. Articles were selected that adopt the terms of use and privacy policies of online social networks as the object of study. Results: From this corpus, it was found that the topic of personal data privacy on online social networks has been approached under several aspects, and especially in relation to the treatment of the digital legacy. Conclusions: This theme has achieved increasing prominence and has been shown to be recurrent, relevant and current, probably due to the large volume of data captured and stored by the application providers.
\end{abstract}

Descriptors: Privacy. Personal data. Terms of Use. Privacy Policy. Online Social Networks.

\section{TÉRMINOS DE USO Y POLÍTICA DE PRIVACIDAD PARA REDES SOCIALES EN LÍNEA}

\begin{abstract}
RESUMEN
Objetivo: Insertado en el debate contemporáneo sobre la privacidad de los datos personales y las políticas de información, este artículo tiene como objetivo discutir cómo las publicaciones científicas brasileñas han abordado los términos de uso y las políticas de privacidad de las redes sociales en línea. Adicionalmente, tiene como objetivo identificar qué aspectos se han abordado en la literatura académica, en relación a estos términos de uso y las respectivas políticas de privacidad. Metodología: Se realizó un estudio exploratorio, analítico-descriptivo, con una revisión sistemática de la literatura de los artículos publicados en revistas brasileñas y actas de congresos disponibles en Portal Capes. Se seleccionaron artículos que adoptan los términos de uso y políticas de privacidad de las redes sociales online como objeto de estudio. Resultados: A partir de este corpus, se encontró que el tema de la privacidad de los datos personales en las redes sociales en línea ha sido abordado bajo varios aspectos, y especialmente en relación al tratamiento del legado digital. Conclusiones: Este tema ha ganado cada vez más importancia y se ha demostrado que es recurrente, relevante y actual, probablemente debido al gran volumen de datos capturados y almacenados por los proveedores de aplicaciones.
\end{abstract}


Descriptores: Privacidad. Datos personales. Condiciones de uso. Políticas de privacidad. Redes sociales online.

Recebido em: 30.07.2021

Aceito em: 17.12.2021 